11 a 13 de setembro de 2019 - Universidade de Brasília UnB

\title{
O POTENCIAL DO DATA MINING PARA TRATAMENTO DA COMPLEXIDADE NO CONTEXTO DO GERENCIAMENTO HÍBRIDO DE PROJETOS DE NOVOS PRODUTOS
}

Michael Jordan Bianchi (michael_bianchi@usp.br) - Departamento de Engenharia de Produção / Escola de Engenharia de São Carlos / Universidade de São Paulo

Daniel Capaldo Amaral (amaral@usp.br) - Departamento de Engenharia de Produção / Escola de Engenharia de São Carlos / Universidade de São Paulo

\section{RESUMO}

Sabe-se que quantidades significativas de dados são coletadas e armazenadas em ambientes de gestão de projetos em função do uso de tecnologias digitais de comunicação e armazenamento de dados. Ao mesmo tempo, tem-se o desafio de gerenciar projetos cada vez mais complexos em ambientes que requerem níveis significativos de agilidade. Uma das formas de lidar com o problema é por meio dos modelos híbridos de gestão. Será que a mineração de dados, utilizada para descobrir conhecimentos a partir de grandes quantidades de dados, poderia auxiliar no desenvolvimento de modelos híbridos, permitindo que as organizações lidem com a complexidade de seus projetos? O presente estudo identificou o estado da arte em relação ao uso de mineração de dados em gestão de projetos visando responder essa questão. Dentre as técnicas de data mining priorizaram-se as regras de associação, as quais visam encontrar padrões interessantes em grandes conjuntos de dados. Por meio de uma revisão bibliográfica sistemática foram encontrados dez estudos que propõe o uso de regras de associação em gestão de projetos. A pesquisa constata o uso de data mining em gestão de projetos e indica potenciais usos dessa técnica para tratar a complexidade no contexto do gerenciamento híbrido de projetos, sendo a combinação de práticas e sua relação com o desempenho do projeto os mais evidenciados.

Palavras chave: Data mining; Regras de Associação, Modelos Híbridos, Gerenciamento de projetos. 


\section{INTRODUÇÃO}

O gerenciamento de projetos complexos e em ambientes dinâmicos será a marca da nova indústria 4.0 e da inovação por meio de ecossistemas (IANSITI; LAKHANI, 2014). O resultado são projetos cada vez mais complexos devido às mudanças tecnológicas, diversidade de atores, modelos de negócios, enfim, ao novo ambiente de negócios (SAN CRISTÓBAL et al., 2019). Essa complexidade está relacionada a fatores como o número de variáveis/componentes e a interação entre eles (LUO et al., 2017).

Imani, Nakano, Anantatmula, (2017) defendem a combinação de práticas de gestão como uma alternativa para lidar com projetos desse tipo, por terem a capacidade de serem mais adaptáveis à essas necessidades inerentes. Trata-se dos chamados modelos híbridos, um dos temas mais discutidos na pesquisa sobre agilidade e projetos de inovação (ADELAKUN et al., 2017; CIRIC et al., 2018).

Várias propostas de modelos híbridos podem ser encontradas na literatura, como o de Cooper (2014), Sommer et al., (2015) e Conforto e Amaral (2016). Todavia, esses modelos possuem a limitação de terem sido criados em ambientes específicos e para determinados tipos de projetos e indústrias, dificultando seu uso para quaisquer tipos de projeto. Um dos principais desafios é desenvolver habilidades e ferramentas capazes de lidar com à complexidade e dinamismo e também adaptar as práticas de gestão de projetos às necessidades do projeto.

A mineração de dados é uma das estratégias possíveis para problemas desta natureza. A aplicação de algoritmos de mineração de dados em gerenciamento de projetos pode ser viável para descobrir padrões e conhecimentos interessantes a partir de grande quantidade de dados, a fim de auxiliar na tomada de decisões. Uma das principais técnicas para a identificação desses padrões é a chamada regra de associação. Esse estudo propõe uma revisão bibliográfica sobre regras de associação em gestão de projetos, a fim de identificar o estado da arte sobre o tema e buscar estudos que auxiliem as empresas a lidarem com a complexidade no contexto de gerenciamento híbrido de projetos. 


\section{REVISÃO TEÓRICA}

Este capítulo apresenta os conceitos fundamentais que orientaram o desenvolvimento da presente pesquisa.

\subsubsection{Mineração de dados (Data mining) e regras de associação}

A informatização da sociedade e da tecnologia, a coleta e armazenamento de informações evoluiu ao longo dos anos, exigindo o uso de técnicas apropriadas para descobrir informações valiosas e transformá-las em conhecimento ( HAN et al., 2009; HAN; KAMBER; PEI, 2012).

Esses fatos culminaram no surgimento da mineração de dados, que pode ser definida como o processo de descobrir padrões e conhecimentos interessantes a partir de grandes quantidades de dados, sendo aplicada nas mais variadas áreas e setores da economia (HAN; KAMBER; PEI, 2012).

Uma técnica que se destaca na mineração de padrões frequentes são as regras de associação (association rules). Ela permite encontrar padrões interessantes (relacionamentos, dependências) em grandes conjuntos de itens de dados (CIOS et al., 2007). Essa associação entre itens pode fornecer informações importantes para o usuário. Dois exemplos clássicos são fornecidos por Cios et al., (2007):

\section{Compra ( $x$, leite_desnatado) $\Rightarrow$ compra ( $x$, pão_branco) $[s=2.5 \%, c=60.0 \%]$ \\ II. Formação (x, Engenharia_da_Computação) AND realiza_curso (x, Análise Avançada de Dados e Tomada de Decisão) $\Rightarrow$ nível $(x, P h D)[s=1 \%, c=75 \%]$}

X é uma variável representando o consumidor/pessoa. A parte da esquerda é chamada de antecedente e a da direita de consequente. A primeira regra afirma que a pessoa que compra leite desnatado também acaba comprando pão branco com um suporte de 2,5\% e confiança de $60 \%$. A segunda regra afirma que os alunos graduados em Engenharia da Computação e que cursam Análise Avançada de Dados e Tomada de Decisão estão no nível de doutorado. com suporte de $1 \%$ e confiança de $75 \%$.

O suporte da regra indica a frequência (probabilidade com que esse padrão aparece na base de dados), sendo a razão do número de transações contendo A e B juntos pelo número total de transações (CIOS et al., 2007). A confiança indica a força de implicação na regra, sendo a 
relação do número de transações contendo A e $\mathrm{B}$ com o número de transações contendo $\mathrm{A}$ (CIOS et al., 2007).

Imagine uma empresa, ou um conjunto de empresas, registrando informações a respeito de seus projetos, como práticas utilizadas, barreiras encontradas, soluções acatadas, entre outros. Quais tipos de padrões e informações poderiam ser revelados a partir desses dados utilizando técnicas de mineração de dados? É possível pensar em padrões de práticas de gestão de acordo com as características e ambiente do projeto? Essas informações poderiam auxiliar na criação de modelos de gestão? E no apoio ao gerenciamento? O presente estudo visa identificar o estado esse tema, analisando o potencial uso da mineração de dados para lidar com a complexidade no contexto do gerenciamento híbrido de projetos.

\subsection{Modelos híbridos de gestão}

Modelos híbridos de gestão estão sendo cada vez mais estudados e utilizados por diferentes indústrias. Esse interesse é impulsionado pela necessidade de lidar com os novos desafios do mercado, como complexidade e inovação, e diferentes tipos de projetos em uma mesma organização. Nesses casos, as abordagens puras de gestão (ágil e tradicional) não suprem totalmente as necessidades impostas.

Utilizaremos aqui a definição proposta por Conforto et al., (2015) que definem modelos híbridos como:

\footnotetext{
“A combinação de princípios, práticas, técnicas e ferramentas de diferentes abordagens em um processo sistemático que visa adequar a gestão para o contexto de negócio e tipo específico de projetos. Têm como objetivo maximizar o desempenho do projeto e produto, proporcionar um equilíbrio entre previsibilidade e flexibilidade, reduzir os riscos e aumentar a inovação, para entregar melhores resultados de negócio e valor agregado para o cliente."
}

Existe uma ampla gama de propostas de modelos híbridos na literatura. Citamos aqui para fins de exemplo, o XPrince (NAWROCKI et al., 2006), V-model +Scrum (ANITHA; SAVIO, 2013), Agile-Stage-Gate (COOPER, 2014), Industrial Scrum Framework (SOMMER et al., 2015), e o IVPM2 (CONFORTO; AMARAL, 2016). Apesar da existência desses modelos, todos apresentam a limitação de terem sido desenvolvidos para um contexto especifico, ou seja, para as particularidades do negócio, características do produto, cliente, mercado e tecnologia 
especificas. Por exemplo, o XPrince foi proposto para diferentes tipos de software, enquanto o IVMP2 é voltado para a manufatura. Por esse motivo eles não devem ser generalizados para serem utilizados em qualquer tipo de projeto ou indústria.

O quadro abaixo elenca os principais diferenciais e problemas relacionados aos modelos híbridos de gerenciamento de projetos.

Quadro 1. Diferenciais e problemas dos atuais modelos híbridos de gestão

\begin{tabular}{|c|c|}
\hline Diferenciais (D) & Problemas $(\mathbf{P})$ \\
\hline $\begin{array}{l}\text { 1. Combinam princípios, práticas, técnicas ou } \\
\text { ferramentas de duas ou mais abordagens; } \\
\text { 2. Customizados para atender às especificidades } \\
\text { do tipo de projeto; } \\
\text { 3. Equilibram previsibilidade, antecipação e } \\
\text { minimização de riscos com flexibilidade; } \\
\text { 4. eliminação de atividades e documentação que } \\
\text { não adicionam valor para a gestão do projeto; } \\
\text { 5. Podem apresentar diferentes papéis e } \\
\text { responsabilidades trabalhando de forma } \\
\text { colaborativa; } \\
\text { 6. Combinam disciplina de processos com } \\
\text { autogestão das equipes. }\end{array}$ & $\begin{array}{l}\text { 1. Desenvolvidos para um contexto especifico, e não } \\
\text { devem ser generalizados para qualquer tipo de projeto; } \\
\text { 2. Falta de um procedimento sistemático indicando } \\
\text { como combinar as práticas; } \\
\text { 3. Não há definição de quais fatores devem ser } \\
\text { considerados para desenvolver modelos híbridos; } \\
\text { 4. Não há uma recomendação de práticas para } \\
\text { diferentes projetos e setores da economia; } \\
\text { 5. Não há análise de desempenho das práticas } \\
\text { utilizadas; } \\
\text { 6. Não há associação de riscos por tipo de projeto; } \\
\text { 7. Não há associação de problemas ou lições aprendidas } \\
\text { com práticas. }\end{array}$ \\
\hline
\end{tabular}

Fonte: Baseado em Conforto et al., (2015) e Bianchi (2017).

Será possível que as técnicas de mineração de dados podem ajudar os pesquisadores e profissionais da área a superarem esses desafios? A presente pesquisa traz uma revisão dos estudos envolvendo gestão de projetos e mineração de dados para tentar responder essa questão e propor novas soluções.

\section{METODOLOGIA}

O estudo compreendeu a realização de uma revisão bibliográfica sistemática (RBS) a fim de identificar estudos que envolviam o uso de regras de associação no contexto de gestão de projetos, a fim de verificar o potencial do data mining para auxiliar o gerenciamento de projetos. Foi utilizado o método de RBS proposto por Conforto, Amaral e Silva (2011). As etapas do estudo podem ser verificadas no QUADRO 2. 
QUADRO 2 - Etapas de desenvolvimento do estudo.

\begin{tabular}{|c|c|}
\hline Etapa 1 & $\begin{array}{l}\text { Inicialmente foram definidos o problema de pesquisa, o objetivo da RBS, os critérios para inclusão } \\
\text { de artigos no estudo, as fontes primárias de informações, as strings de busca e o cronograma da } \\
\text { RBS. }\end{array}$ \\
\hline Etapa 2 & $\begin{array}{l}\text { As busca foram coduzidas nas bases de dados pré-estabelecidas (Web of Science }{ }^{\circledR} \text { e Google } \\
\text { Scholar) utilizando as strings de busca definidas na etapa anterior. Vale ressaltar a necessidade de } \\
\text { uma atenção voltada para os operadores lógicos utilizados nas bases de dados. Um total de } 1101 \\
\text { artigos foram encontrados }\end{array}$ \\
\hline Etapa 3 & $\begin{array}{l}\text { Realizadas as buscas, os resultados passaram pelos três filtros propostos. O primeiro filtro envolveu } \\
\text { a leitura do título, resumo e palavras-chave do trabalho, as quais deviam estar em concordância } \\
\text { com as utilizadas nas strings de busca e critério preestabelecidos, culminando em } 50 \text { trabalhos } \\
\text { selecionados. No segundo filtro foi realizado a leitura da introdução e conclusão desses artigos, e } \\
15 \text { passaram para o proximo filtro. O terceiro filtro consistiu na leitura completa dos artigos, tendo } \\
\text { como resultado final } 11 \text { artigos selecionados. }\end{array}$ \\
\hline Etapa 4 & $\begin{array}{l}\text { Foi realizada a busca cruzada, identificando trabalhos relevantes a partir das citações dos autores } \\
\text { nos artigos selecionados. }\end{array}$ \\
\hline Etapa 5 & Consistiu em catalogar e armazenar os artigos selecionados em um repositório de artigos. \\
\hline
\end{tabular}

As strings de busca utilizadas foram: "Association Rules" or "Association analysis" or "Apriori Algorithm" AND Project* or "Project management" or "Project assessment" or "Agile" or "Plan-driven" or "warterfall". Foi necessário realizar a exclusão dos resultados voltados para a área da saúde, uma vez que muitos trabalhos relacionados a medicina e diagnóstico utilizam regras de associação.

Os critérios de seleção dos artigos foram: 1) Descrever sobre aplicações de regras de associação na área de gestão de projetos; 2) Descrever sobre o uso de regras de associação na escolha de práticas de gestão de projetos; e 3) Relacionar o uso de regras de associação na configuração de modelos de gestão de projetos.

\section{RESULTADOS E DISCUSSÃO}

A revisão bibliográfica sistemática permitiu identificar diversos autores que têm estudado o uso de técnicas de mineração de dados na área de gerenciamento de projetos. No Quadro 3 apresentamos os estudos encontrados na literatura que discutem o uso de regras de associação em gestão de projetos.

A maioria dos trabalhos apresentados focam na aplicação de modelos matemáticos, sem avaliar o impacto das técnicas no desempenho ou prática do gerenciamento de projetos, não sendo 
explorados com a devida atenção como forma de ajudar efetivamente o gerente de projetos ou profissional da área na condução de seus projetos.

Apesar das limitações mencionadas, destaca-se que os autores concordam no potencial uso de técnicas de data mining para aperfeiçoar e auxiliar na evolução da área de gerenciamento de projetos, seja para alocar recursos, estimar esforço e tempo para coletar uma atividade do projeto, ou identificar os fatores de sucesso de um determinado projeto.

Exploramos a seguir esse potencial, relacionando os problemas referentes a modelos híbridos destacados na seção 2.2 e como a mineração de dados poderia auxiliar na resolução desses desafios.

\section{P1. Desenvolvidos para um contexto especifico, e não devem ser generalizados para qualquer}

tipo de projeto. As práticas de modelos híbridos precisam ser personalizadas para contextos específicos, sem generalização para projetos da organização ou por tipos. O uso de mineração de dados pode auxiliar nessa questão, identificando a partir de projetos passados, quais práticas de um determinado modelo de gestão são benéficas a serem utilizados naquele contexto e tipo de projeto, e quais precisarão ser revistas. Dessa forma as organizações podem se inspirar em um modelo híbrido existente, adaptando-o para sua realidade.

\section{P2. Falta de um procedimento sistemático indicando como combinar as práticas e P4. Não} há uma recomendação de práticas para diferentes projetos e setores da economia. A mineração de dados tem o potencial de analisar grandes volumes de dados de projetos, e a partir desses dados utilizar algoritmos capazes de relacionar práticas de gestão com o ambiente organizacional, características de um projeto e nível de agilidade, a fim de recomendar um modelo de gestão. Assim, automatiza-se o procedimento de combinação de práticas de gestão, podendo identificar as práticas mais alinhadas com o setor da empresa e projetos que essa desenvolve, eliminando a necessidade de um especialista em gestão de projetos para tal. 


\section{de Desenvolvimento do Produto}

11 a 13 de setembro de 2019 - Universidade de Brasília UnB

\begin{tabular}{|c|c|}
\hline Autor & \\
\hline $\begin{array}{l}\text { Chawla, Arunasalam } \\
\text { and Davis (2003) }\end{array}$ & $\begin{array}{l}\text { Utilizaram association rules networks (ARNs) em conjunto com a técnica de cluster para descobrir padrões que caracterizassem o sucesso ou } \\
\text { fracasso dos projetos do tipo Open Source Software (OSS), retirados do SourceForge.net. }\end{array}$ \\
\hline Veloso (2003) & $\begin{array}{l}\text { Usaram regras de associação para auxiliar a escolha de recursos para formar a equipe de projeto. Esta atividade lida com diversas variáveis, } \\
\text { como características técnicas e pessoais, disponibilidade; características do projeto e do cliente; entre outras. As regras propostas pelo autor são } \\
\text { do tipo: se A trabalhar num determinado projeto, então B tem x\% de probabilidade de trabalhar nesse mesmo projeto. }\end{array}$ \\
\hline García et al., (2004) & $\begin{array}{l}\text { Utilizaram algoritmos de regras de associação para descobrir padrões que relacionam o tamanho de um projeto de software, com outros } \\
\text { atributos, como o esforço necessário para realizá-lo. O objetivo foi encontrar padrões para auxiliar nas estimativas de tamanho do projeto } \\
\text { permitindo um bom planejamento e redução de custos. }\end{array}$ \\
\hline Song et al., (2006) & $\begin{array}{l}\text { Integraram a técnica de regras de associação com a de classificação para prever defeitos em softwares e os esforços necessários para a correção } \\
\text { desses, a partir de dados históricos de engenharia de software. É possível entender como um defeito se relaciona com outro, possibilitando que } \\
\text { o gerente de projetos tome decisões e evites defeitos ou problemas futuros. }\end{array}$ \\
\hline García et al., (2008) & $\begin{array}{l}\text { Mineraram dados de projetos por meio de regras de associação para estimar a influência de certos fatores da política de gerenciamento em } \\
\text { vários atributos do projeto de software como fatores tecnológicos, de produto e de processo. Como resultado os autores apresentam onze regras } \\
\text { associando o impacto das políticas de gestão com a qualidade, tempo e esforço de desenvolvimento de softwares. }\end{array}$ \\
\hline $\begin{array}{l}\text { Prasad, Arsiwala and } \\
\text { Singh (2010) }\end{array}$ & $\begin{array}{l}\text { Descobriram fatores que orientam o projeto para o sucesso utilizando regras de associação (Apriori), árvore de decisão, redes neurais e Naive } \\
\text { Bayes. A base de dados foi formada através de um survey on-line, analisando fatores de sucesso e fracasso, além de constatar se o projeto do } \\
\text { respondente foi um sucesso ou não. }\end{array}$ \\
\hline $\begin{array}{l}\text { Azzeh, Cowling e } \\
\text { Neagu (2010) }\end{array}$ & $\begin{array}{l}\text { Utilizam regras de associação e uma teoria chamada "Fuzzy set theory" para predizer o esforço necessário para um determinado estágio do } \\
\text { projeto tomando como base informações de estágios anteriores, a fim de evitar que o plano inicial possua uma grande diferença em relação ao } \\
\text { final. O intuito é permitir que o gerente de projeto realoque o número correto de recursos para terminar no prazo e dentro do orçamento. }\end{array}$ \\
\hline Emanuel et al., (2010) & $\begin{array}{l}\text { Usam as regras de associação para encontrar os fatores de sucesso de Projetos de Software de Código Aberto (OSS) no portal sourceforge.net. } \\
\text { Para os autores o projeto seria um sucesso baseado no número de downloads na plataforma analisada. O trabalho resultou em seis fatores que } \\
\text { devem ser utilizados em projetos desse tipo para aumentar sua probabilidade de sucesso. }\end{array}$ \\
\hline Parsanejad (2013) & $\begin{array}{l}\text { Usam as regras de associação para descobrir a relação/ padrões entre nove critérios de sucesso de projeto, apresentado os resultados } \\
\text { encontrados. Os critérios utilizados são: Custo, Tempo, Qualidade, Satisfação do Cliente/Proprietário/Patrocinador, Satisfação da organização } \\
\text { de gerenciamento de projetos, Satisfação dos integrantes da equipe de projeto, satisfação do fornecedor, satisfação do usuário final, e satisfação } \\
\text { de outras partes interessadas. }\end{array}$ \\
\hline Savchuk et al., (2017) & $\begin{array}{l}\text { Investigaram o uso de regras associativas na fase de testes no desenvolvimento de um software, com o intuito de prever o tempo necessário } \\
\text { para corrigir um determinado erro, através da relação bugs e tempo, além de poder prever o profissional que irá corrigir esse erro de acordo } \\
\text { com suas habilidades. O autor utilizou os dados de dois grandes projetos de código aberto para a pesquisa. }\end{array}$ \\
\hline
\end{tabular}




\section{Congresso Brasileiro de Inovação e Gestão}

\section{de Desenvolvimento do Produto}

\section{1 a 13 de setembro de 2019 - Universidade de Brasília UnB}

\section{P3. Não há definição de quais fatores devem ser considerados para desenvolver modelos}

híbridos. Esse problema deve ser melhor investigado por meio de técnicas apropriadas de correlação e modelagem de dados. Fatores internos e externos à organização que impactam na escolha das práticas a fim de permitir a criação de modelos de gestão.

P5. Não há análise de desempenho das práticas utilizadas. Podemos minerar os dados referentes aos resultados obtidos com as práticas utilizadas em projetos passados, e comparálos com os resultados alcançados com as novas práticas/modelo de gestão utilizado. Aqui também há outra oportunidade de desenvolvimento e discussão. No gerenciamento tradicional aplicam-se métodos como Análise do Valor Agregado para acompanhar a evolução do projeto. No ambiente híbrido podemos incluir equipes ágeis como responsáveis por entregas. Seria possível utilizar técnicas de mineração para combinar dados das equipes ágeis em projetos híbridos e associar com indicadores de valor agregado?

P6. Não há associação de riscos por tipo de projeto. As empresas podem utilizar a mineração de dados para associar riscos potenciais de acordo com o tipo de projeto, possibilitando um gerenciamento de riscos mais consistente e que pode ser aproveitado em projetos futuros.

P7. Não há associação de problemas ou lições aprendidas com práticas. A mineração de dados pode gerar uma lista relacionando os problemas enfrentados nos projetos passados das empresas e como foram superados, sintetizando as lições apreendidas para serem utilizadas em projetos futuros.

\section{CONCLUSÃO}

Modelos híbridos de gestão têm se mostrado interessantes para lidar com essa nova realidade. A combinação de práticas alinhadas com o ambiente organizacional permite customizar o modelo de gestão para a realidade de cada projeto. Entretanto uma série de problemas emerge nesse cenário, como por exemplo encontrar a melhor combinação para cada situação.

A presente pesquisa buscou identificar o potencial uso da mineração de dados em gestão de projetos a fim de auxiliar na resolução desses problemas. Uma análise da literatura permitiu identificar estudos que utilizaram regras de associação para auxiliar na gestão de seus projetos, seja para auxiliar a escolha de recursos para formar a equipe de projeto, para descobrir o esforço 
necessário para realizar um projeto ou partes dele, prever defeitos em softwares e os esforços necessários para a correção desses. Esses estudos revelam o potencial uso da mineração de dados em gestão projetos.

Baseado nos resultados encontrados o presente estudo propôs o uso de mineração de dados, mais especificamente as regras de associação, para solucionar os problemas relacionados com a aplicação de modelos híbridos de gestão. Ao todo sete problemas foram abordados visando auxiliar as empresas e profissionais a encontrar a combinação de práticas mais apropriadas para seus projetos de acordo com suas especificidades. Os problemas de combinação de práticas e desempenho parecem os mais promissores.

\section{AGRADECIMENTOS}

O presente trabalho foi realizado com apoio da Coordenação de Aperfeiçoamento de Pessoal de Nível Superior - Brasil (CAPES) - Escola de Engenharia de São Carlos - Programa de PósGraduação em Engenharia de Produção - Código de Financiamento 001.

\section{REFERÊNCIAS}

ADELAKUN, Olayele et al. Hybrid Project Management : Agile with Discipline. 2017.

ANITHA, P. C.; SAVIO, Deepti. Managing Requirements Volatility while ' Scrumming' within the V-Model. 2013 3rd International Workshop on Empirical Requirements Engineering (EmpiRE), p. 17-23, 2013.

AZZEH, Mohammad; COWLING, Peter I.; NEAGU, Daniel. Software Stage-Effort Estimation Based on Association Rule Mining and Fuzzy Set Theory. 2010 10th IEEE International Conference on Computer and Information Technology, p. 249-256, 2010.

CHAWLA, Sanjay; ARUNASALAM, Bavani; DAVIS, Joseph. Mining open source software (oss) data using association rules network. Advances in Knowledge Discovery and Data Mining, p. 564-564, 2003.

CIOS, K. J. et al. Data Mining A Knowledge Discovery Approach. Springer Science \& Business Media, 2007.

CIRIC, Danijela et al. Agile Project Management in New Product Development and Innovation Processes : Challenges and Benefits Beyond Software Domain. 2018 IEEE International Symposium on Innovation and Entrepreneurship (TEMS-ISIE), p. 1-9, 2018.

CONFORTO, E. et al. Modelos híbridos unindo complexidade, agilidade e inovação. Revista Mundo PM, v. 64, p. 10-17, 2015.

CONFORTO, E. C.; AMARAL, D. C. Agile project management and stage-gate model—A hybrid framework for technology-based companies. Journal of Engineering and Technology Management, v. 40, p. 1-14, 2016.

COOPER, Robert G. What's Next?: After Stage-Gate. Research-Technology Management, v. 57, n. 1, p. 20$31,2014$.

EMANUEL, Andi Wahju Rahardjo et al. Success factors of OSS projects from sourceforge using Datamining Association Rule. 2010 International Conference on Distributed Frameworks for Multimedia Applications, 


\section{p. 1-8, 2010.}

GARCÍA, María N. et al. An association rule mining method for estimating the impact of project management policies on software quality, development time and effort. Expert Systems with Applications, v. 34, n. 1, p. 522529,2008

GARCÍA, María N. Moren. et al. Building knowledge discovery-driven models for decision support in project management. Decision Support Systems, v. 38, n. 2, p. 305-317, 2004.

HAIR, Joseph et al. Análise multivariada de dados. 6. ed. ed. Porto Alegre: Bookman, 2009.

HAN, Jiawei; KAMBER, Micheline; PEI, Jian. Data Mining: Concepts and Techniques. Morgan Kaufmann, 2012.

IANSITI, Marco; LAKHANI, Karim R. How Connections, Sensors, and Data Are Revolutionizing Business. Harvard Business Review, n. November 2014, 2014.

IMANI, Takeomi; NAKANO, Masaru; ANANTATMULA, Vittal. Does a Hybrid Approach of Agile and PlanDriven Methods Work Better for IT System Development Projects? Int. Journal of Engineering Research and Application, n. March 2017, p. 39-46, 2017.

LUO, Lan et al. Construction Project Complexity : Research Trends and Implications. v. 143, n. 7, 2017.

NAWROCKI, Jerzy et al. Balancing agility and discipline with XPrince. Lecture Notes in Computer Science, p. 266-277, 2006.

PARSANEJAD, Mohammadreza. Applying Association Rules to Explore Relationships among Project Success Criteria. Journal of Industrial and Intelligent Information, v. 1, n. 2, p. 77-80, 2013.

PRASAD, Anand; ARSIWALA, Juzer; SINGH, Praval Pratap. Estimating and improving the probability of success of a software project by analysing the factors involved using data mining. Proceedings - 2010 International Conference on Artificial Intelligence and Education, ICAIE 2010, n. 1, p. 391-394, 2010.

SAN CRISTÓBAL, J. R. et al. Complexity and Project Management: Challenges, Opportunities , and Future Research. Complexity, 2019.

SAVCHUK, Tamara O. et al. The technology of searching the associative rules while developing the software. $\mathrm{n}$. August 2017, p. 104451Y, 2017.

SOMMER, Anita Friis et al. Improved Product Development Performance through Agile/Stage-Gate Hybrids: The Next-Generation Stage-Gate Process? Research Technology Management, v. 58, n. 1, p. 34-44, 2015.

SONG, Qinbao et al. Software defect association mining and defect correction effort prediction. IEEE Transactions on Software Engineering, v. 32, n. 2, p. 69-82, 2006.

VELOSO, M. J. S. A. Regras de associação aplicadas a um método de apoio ao planejamento de recursos humanos. 2003. Universidade do Porto, 2003. 